\title{
Efficiency of arrhythmia detection by nurses in a coronary care unit using a decentralised monitoring system ${ }^{1}$
}

\author{
STIG HOLMBERG, LARS RYDEN², AND ANDERS WALDENSTRÖM \\ From the Department of Medicine I, Division of Cardiology, Sahlgren's Hospital, S-413 45 Göteborg, \\ Sweden
}

The efficiency of nurses in detecting arrhythmias was studied in a coronary care unit (CCU) with 6 beds, equipped with a system for decentralised electrocardiographic display and with well-defined routines for arrhythmia documentation. Arrhythmias were classified and reported at half-hour intervals. Fifty patients were included in the study. A continuous electrocardiographic recording was subsequently analysed beat by beat and used as reference.

The arrhythmias were classified into: (i) 'benign' arrhythmias, (ii) 'warning' arrhythmias, (iii) 'malignant' arrhythmias, and (iv) ventricular tachycardia.

The detection rate for different arrhythmias varied from 96 per cent for patients with 'benign' arrhythmias occurring in at least three 1-minute periods per half-hour and 86 per cent for patients with 'warning' arrhythmias, to 42 per cent for patients with ventricular tachycardia.

Special attention was paid to infrequently occurring arrhythmias which were analysed in relation to half-hour periods. Of 'warning' arrhythmias occurring during only one 1-minute period per half-hour, 48 per cent were detected, but the detection rate increased to 71 per cent when the same type of arrhythmia occurred during two or more 1-minute periods per half-hour.

The mean delay time for detection of 'warning' and 'malignant' arrhythmias was less than half an hour. The exact delay time could not be evaluated as the study was based on the half-hourly reports of the nurses. The fact that the most infrequent 'warning' arrhythmias were detected in 48 per cent during the first possible minute indicates, however, that the true delay time is much shorter.

The efficiency of arrhythmia detection is higher in this than in other published studies, both for detection rate and delay time. Possible explanations for this are that well-defined routines for arrhythmia documentation are established and that a system for decentralised electrocardiographic display is used.

The introduction of coronary care units (CCU) led to a substantial decrease in hospital mortality in acute myocardial infarction (Christiansen et al., 1970; Hofvendahl, 1971). Originally centred on resuscitation, the main aim of the CCUs gradually changed to early treatment of ventricular arrhythmias in order to prevent deaths from ventricular fibrillation (Meltzer and Kitchell, 1972). This made continuous electrocardiographic monitoring and early detection of ventricular arrhythmias imperative. Most CCUs still rely upon nurses or other specially trained staff for the observation and inter-

1This study was supported by a grant from the Swedish National Association against Heart and Chest Diseases.

'Present address: Kărnsjukhuset S-54100 Sköude, Sweden.

Received for publication 13 September 1976 pretation of the electrocardiogram. Despite 10 years experience and world-wide application of the principles of coronary care, surprisingly little is known about the accuracy of arrhythmia detection in the CCU. The detection of ventricular arrhythmias has probably been assumed to be adequate, but, recent data from some CCUs have revealed a surprisingly low detection rate of arrhythmias (Mogensen, 1970; Romhilt et al., 1973; Vetter and Julian, 1975).

The equipment, staff, work routines, and training programmes vary considerably between different CCUs. These variations may be expected to have a profound effect on the accuracy of arrhythmia detection. This subject, however, has been studied very little. No conclusions can be drawn about the 
importance of such factors until systematic studies have been performed in CCUs of various designs and modes of operation. The aim of the present study was to obtain detailed information on the detection of ventricular arrhythmias in our CCU. For this purpose a continuous electrocardiographic record was compared with arrhythmia detection by nurses.

\section{Subjects and methods}

CORONARY CARE UNIT-

EQUIPMENT AND ORGANISATION

The CCU consists of 6 beds and is part of a cardiac unit consisting of 24 beds. The unit is small and has space only for a small desk for the nurses and a limited area for preparation of drugs and storage of the most essential equipment. A special system for decentralised presentation of the electrocardiogram has been developed and used in this CCU (Medivision, NUKAB, Göteborg, Sweden). Briefly, the electrocardiographic signal from each of the 6 patients is amplified by a conventional amplifier and transformed into a video signal. The system includes a solid state memory which allows presentation of the last five seconds of the electrocardiogram. The signals from all six patients are displayed on TV screens, placed one beside each bed and one on the nurses' desk. Thus, the electrocardiogram from every patient can be monitored on any of the TV screens. This permits the nurses to continue monitoring wherever they are working in the CCU. Furthermore, the electrocardiogram can be continuously recorded on paper at any of the TV screens. The nurses were instructed to start recording as soon as they detected an arrhythmia requiring further analysis.

At least two members of the staff (one nurse and one aide) are always present in the CCU. There are strict rules for the documentation of arrhythmias detected on the monitor. Every half hour all arrhythmias are systematically classified and reported on a standardised coded form (chart available by request to the authors). The absence of arrhythmia is also noted in these record forms.

\section{PATIENTS AND ELECTROCARDIOGRAPHIC RECORDING}

Fifty-two patients admitted to the CCU with verified or suspected acute myocardial infarction were studied. They were all included in a study of the effectiveness of lignocaine in the treatment of ventricular arrhythmias and they all received lignocaine either as an intravenous bolus injection of 75 $\mathrm{mg}$ followed by a constant infusion of $2 \mathrm{mg}$ per minute or as an intramuscular injection of $300 \mathrm{mg}$ into the vastus lateralis muscle. Except for this antiarrhythmic treatment, the patients were managed according to the routine procedures of the CCU (Henning and Holmberg, 1971), and thus the demands on the nurses for arrhythmia detection and documentation were the same before and after lignocaine treatment. A detailed description, including pertinent clinical data of the patients, has been presented elsewhere (Rydén et al., 1973, 1975b). A multichannel ink-writing electrocardiograph with a paper speed of $10 \mathrm{~mm} / \mathrm{s}$ (Mingograf81, Siemens Elema AB, Sweden) was started as soon as the patient received lignocaine and recording was continued for at least 3 hours. This electrocardiographic paper record was used for subsequent arrhythmia analysis and all data concerning the occurrence of ventricular ectopic activity refer to this continuous record, as has been described previously by Mogensen (1970). The continuous electrocardiogram was analysed to determine the presence or absence of ventricular arrhythmias. When ventricular premature contractions (VPC) were present they were classified into the following groups: 1 to $5 \mathrm{VPCs} / \mathrm{min}$; $>5 \mathrm{VPCs} / \mathrm{min}$; $R$ on T VPCs

$$
\text { (i.e. } \left.\frac{R-R^{\prime}}{R-T}<0.85\right) \text {; }
$$

multifocal VPCs; paired VPCs; and ventricular tachycardia (VT), defined as 3 or more VPCs in sequence at the rate of $\geqslant 100$ beats/min.

The reliability of the arrhythmia record forms completed by the nurses was tested by comparison with the analysis of the continuous electrocardiogram for the corresponding half-hour period. The accuracy of the nurse-based detection of ventricular tachyarrhythmias was considered for the following subgroups: (i) 'benign' arrhythmias: 1 to $5 \mathrm{VPCs} / \mathrm{min}$; (ii) 'warning' arrhythmias: $>5 \mathrm{VPCs} / \mathrm{min}$, multifocal VPCs, paired VPCs, R on T VPCs, or VT; (iii) 'malignant' arrhythmias: multifocal VPCs, paired VPCs, $R$ on T VPCs, or VT; (iv) VT.

The nurse-based arrhythmia detection was considered correct if the continuous electrocardiogram and the arrhythmia record forms for the corresponding half-hour periods could be allocated to the same ventricular tachyarrhythmia sub-groups. Following this allocation the data were analysed in two different ways. First, the relation between total and detected ventricular tachyarrhythmias was investigated for each patient (patient-related analysis), and secondly, the same relation was investigated for each half-hour period (time-related analysis). In this latter analysis special attention was devoted to infrequent arrhythmias. These were subdivided into three groups in which there were 
(A) half-hours with only one 1-minute period containing arrhythmias, (B) half-hours with two or three 1-minute periods containing arrhythmias; (C) half-hours with four or five 1-minute periods containing arrhythmias.

The nurses were not informed about the present study.

\section{Results}

Two patients had to be excluded because of lack of their arrhythmia record forms. In the remaining 50 patients, 13 half-hour periods had to be excluded because of lack of parts of arrhythmia record forms and/or uninterpretable electrocardiograms, leaving 287 accepted half-hour periods.

\section{PATIENT-RELATED ANALYSIS}

Benign arrhythmias Thirty-seven of the 50 patients had at least one 1 -minute period containing 1 to 5 VPCs/min. In $26(70 \%)$ of these patients the arrhythmia was detected during the first half-hour in which it occurred. In another 6 patients the arrhythmia was detected later, when there were repeated half-hour periods with episodes of arrhythmia. In 5 patients $(14 \%)$, the arrhythmia was not detected at all by the nurses (Fig. 1).

Twenty-five patients had at least three 1-minute periods containing 1 to $5 \mathrm{VPCs} / \mathrm{min}$ in a half-hour period. Twenty-two $(88 \%)$ of these were detected in the correct half-hour period; another 2 were detected later and 1 was not detected, giving an overall detection rate of 96 per cent.

Warning arrhythmias occurred in at least one 1-minute period in 33 patients and were detected

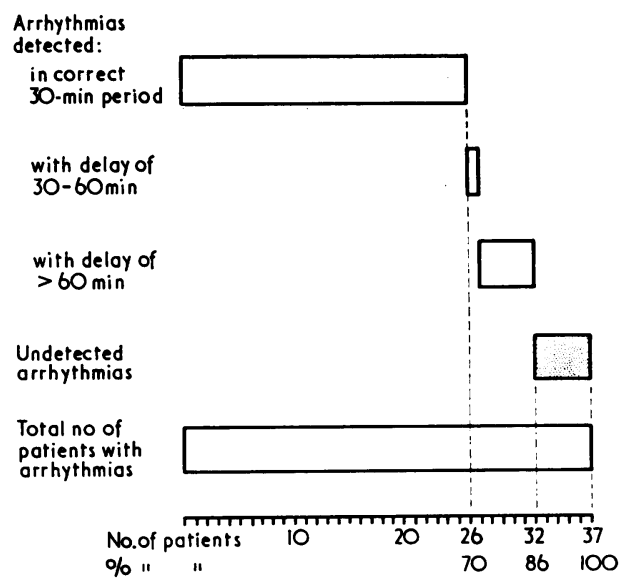

Fig. 1 Detection rate for patients with 'benign' arrhythmias: 1 to 5 VPCs per min. in the first possible half-hour period in $25(76 \%)$. In 3 patients they were detected later, while in 5 they were not detected at all (Fig. 2). When the stricter criterion was applied, 32 patients still had warning arrhythmias, occurring in at least three 1 -minute periods. Of these 28 were detected, giving an overall detection rate of 88 per cent.

Malignant arrhythmias occurred in 30 patients; of these 18 were detected in the first possible halfhour period while one was detected later, giving an overall detection rate of 63 per cent (Fig. 3). By the stricter criterion, 18 patients belonged to this group; fourteen $(78 \%)$ of them were detected.

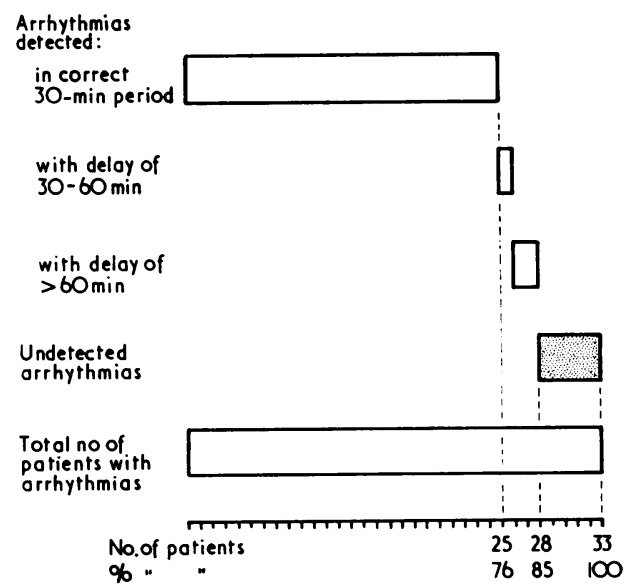

Fig. 2 Detection rate for patients with 'warning' arrhythmias: $>5$ VPCs per min, multifocal VPCs, paired VPCs, $R$ on $T V P C s$, or ventricular tachycardia.

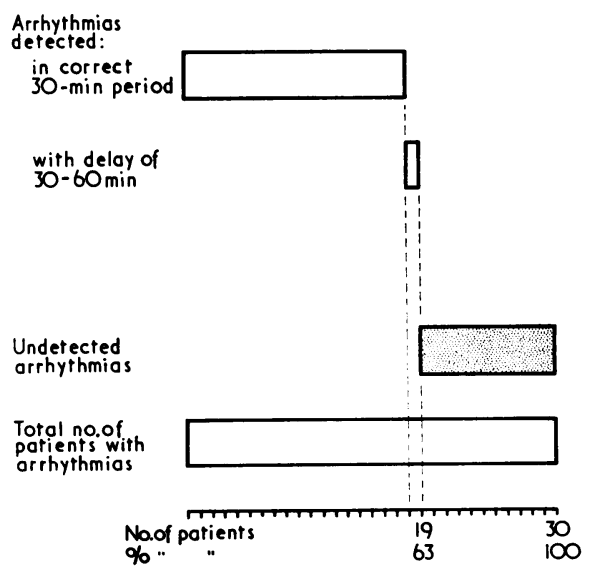

Fig. 3 Detection rate for patients with 'malignant' arrhythmias : multifocal VPCs, paired VPCs, $R$ on $T$ VPCs, or ventricular tachycardia. 
Twelve patients had one or more episodes of ventricular tachycardia. Five $(42 \%)$ were detected, all of them in the first half-hour period (Fig. 4). Five patients had ventricular tachycardia by the stricter criterion; four were detected, all in the first possible half-hour period.

\section{TIME-RELATED ANALYSIS}

Ventricular tachyarrhythmias occurred in 166 (55\%) of the 287 half-hour periods. Benign arrhythmias occurred in 63 half-hour periods and 51 $(81 \%)$ of these were detected by the nurses. Warning arrhythmias occurred in 103 half-hour periods and were detected in $76(74 \%)$ of these. In 85 half-hour periods there were malignant arrhythmias and $53(62 \%)$ were detected. Ventricular tachycardia occurred in 21 half-hour periods, of which $6(29 \%)$ were detected (Fig. 5).

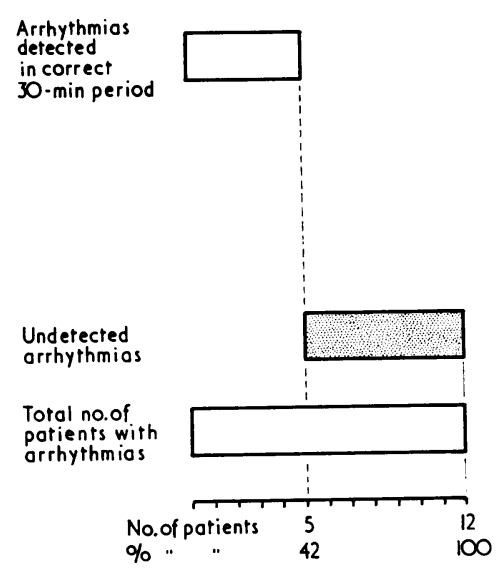

Fig. 4 Detection rate for patients with ventricular tachycardia.

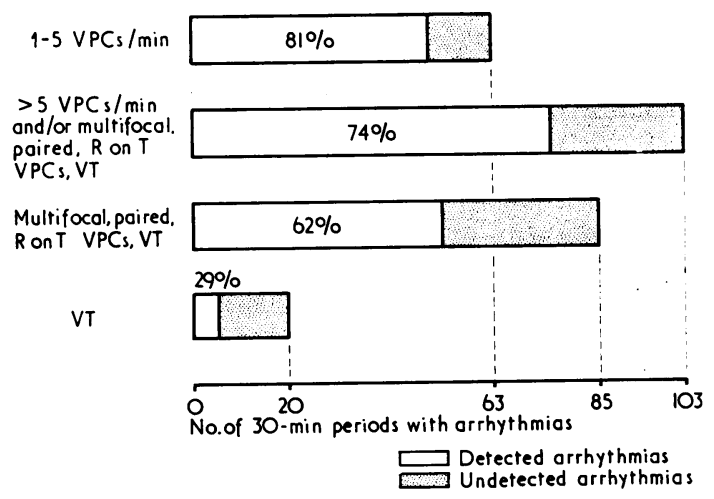

Fig. 5 Detection rate for half-hour periods with different types of arrhythmias.
Infrequent arrhythmias belonging to group A occurred in 52 periods, to group B in 60 periods, and to group $C$ in 15 periods. As can be seen in Fig. 6, the ability of the nurses to detect arrhythmias increases with the number of minutes with arrhythmia within the half-hour period. The figure also shows that the detection rate is different for the various subgroups of ventricular tachyarrhythmias. For example, warning arrhythmias were detected in 48 per cent of cases when the arrhythmia occurred only during one 1-minute period per half-hour. The detection rate increased to 71 per cent when the arrhythmia occurred during two or three 1-minute periods per half-hour.

\section{Discussion}

When studying the reliability of any method of arrhythmia detection the choice of reference is of crucial importance. In the present study a continuous electrocardiographic record was analysed beat by beat by one of the authors and used as reference. This method may be time-consuming but is precise. It was preferred to other methods such as intermittent electrocardiographic sampling and tape recordings with subsequent replay at high speed. These methods are either known to be unreliable (Rydén et al., 1975b) or their accuracy is uncertain. A disadvantage of having a continuous electrocardiographic recording is that the nurses may look at the recording during the investigation. Though it cannot be totally ruled out, there are reasons for believing that this was of minor importance: the nurses were not aware that their accuracy was being tested; they were informed that the continuous electrocardiogram was only for purposes connected with the lignocaine study (Rydén et al., 1973), and that there was no reason to look at the continuous electrocardiogram if no arrhythmias were detected while monitoring the TV screens. Furthermore, as earlier indicated, the nurses routinely use a separate electrocardiographic recorder when they observe an arrhythmia on the TV screen needing further analysis.

The fact that the patients were included in a study of treatment of ventricular arrhythmias could possibly increase the nurses' alertness. However, every patient was already on lignocaine treatment because of previous 'warning arrhythmias'. This is in contrast to the majority of CCU patients where antiarrhythmic treatment has not yet been started and in whom 'warning arrhythmias' have to be looked for. This would decrease rather than increase the alertness of the nurses.

The study shows a high level of efficiency of the nurses in detecting regularly recurring arrhythmias, 
(a)

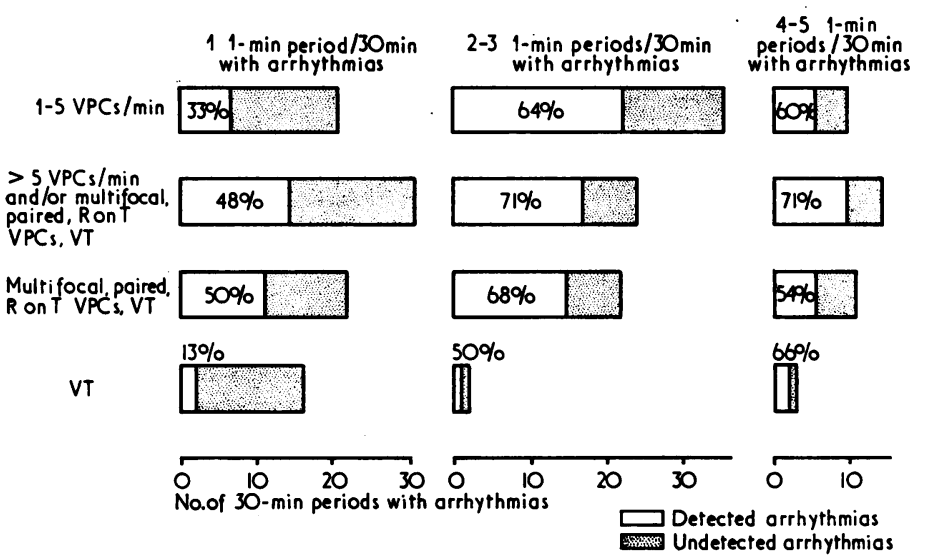

(c)

Fig. 6 Detection rate for infrequent arrhythmias occurring in (a) one 1-min period, (b) two to three 1-min periods, or (c) four to five 1-min periods, during the course of a half-hour period. e.g. 88 per cent of warning arrhythmias occurring during at least three 1-minute periods of a half-hour. The less frequent ventricular tachycardia, however, was detected in only 42 per cent of the cases. In this study, $R$ on T VPCs are not separately analysed because of their low frequency. It could be expected from previous studies (Rydén et al., $1975 \mathrm{~b})$ that the detection rate would be lower than for ventricular tachycardia. Ventricular fibrillation did not occur in any patient during the study and thus provided no indication of underdetection of warning arrhythmias by the nursing staff. The obvious explanation for the great variation in detection rate for different types of ventricular tachyarrhythmias is that the electrocardiograms are not continuously monitored. The supervision can be characterised as a type of intermittent electrocardiographic sampling. With intermittent electrocardiographic sampling, the detection rate varies in a complex manner and is a function of the frequency with which the arrhythmia occurs, the length of the electrocardiographic sample, and the distribution of arrhythmias within the period of observation (Rydén et al., 1975b). The arrhythmia record forms do not give any precise information on the accuracy of detection of infrequently occurring arrhythmias compared with those occurring more frequently. From Fig. 6 it can be calculated that in two-thirds of the half-hour periods containing warning arrhythmias there were no more than 5 arrhythmia-containing 1-minute periods per half-hour. It is interesting to note the high detection rate (48 to 71 per cent) for these infrequent arrhythmias. Though the continuous electrocardiogram was analysed beat to beat, the coding of the content of ventricular tachyarrhythmias was performed on a 1-minute basis. Each minute was classified either as normal or as containing arrhythmias. Therefore, the accuracy of the nurse-based arrhythmia detection within each 1-minute period could not be directly related to variations in the frequency and distribution of the ventricular tachyarrhythmias. It was, however, observed that various types of ventricular tachyarrhythmias occurred with differing frequency and distribution within the 1-minute periods. As an example, ventricular tachycardia consists mostly of 3 to 8 beats in sequence while VPCs often occur repeatedly over the whole 1-minute interval. If the electrocardiographic monitoring of the nurses is continuous within the 1-minute interval all types of ventricular tachyarrhythmias should be detected with the same accuracy. If the nurses' monitoring, on the other hand, is intermittent even within the 1-minute interval the detection of infrequent arrhythmias will be lower. The present results strongly suggest that the monitoring by the nurses is intermittent rather than continuous even within 1-minute periods, since of all half-hour periods with only one arrhythmia-containing 1-minute period ventricular tachycardia was identified in 13 per cent while the rate of detection of warning arrhythmias was 48 per cent.

The reliability of the nurse-based detection of ventricular tachyarrhythmias is higher in our study than in other reports. As can be seen from the Table, both the detection rate and the average delay time for arrhythmia detection compare favourably with those previously published (Romhilt et al., 1973; Vetter and Julian, 1975). The surprisingly high detection rate for ventricular tachycardia in the study by Vetter and Julian (1975) is explained by their use of a rate-dependent alarm system. Thus, the detection of ventricular tachycardia in that study cannot be compared with that in a study relying only on electrocardiographic monitoring by 
Table Efficiency of arrhythmia detection by nurses in three studies

\begin{tabular}{|c|c|c|c|c|}
\hline & & Romhilt et al. (1973) & Vetter and fulian (1975) & Present study \\
\hline & & (31 patients) & (32 patients) & (50 patients) \\
\hline Patients with VPCs & $\begin{array}{l}\text { Total no. of patients } \\
\text { No. detected } \\
\% \text { detected }\end{array}$ & $\begin{array}{l}31 \\
20 \\
65\end{array}$ & $\bar{z}$ & $\begin{array}{l}37 \\
32 \\
86\end{array}$ \\
\hline $\begin{array}{l}\text { Malignant ventricular arrhythmias } \\
\text { (detected within } 30 \mathrm{~min} \text { ) }\end{array}$ & $\begin{array}{l}\text { Total no. of patients } \\
\text { No. detected } \\
\% \text { detected }\end{array}$ & - & $\begin{array}{r}23 \\
4 \\
17\end{array}$ & $\begin{array}{l}30 \\
18 \\
60\end{array}$ \\
\hline Average delay time $(\mathrm{h})$ before detection & $\begin{array}{l}\text { VPCs } \\
\text { Malignant VPCs }\end{array}$ & $\begin{array}{l}18 \\
10\end{array}$ & $5 \cdot 6 \star \star$ & $\begin{array}{l}<0.5 \\
<0.5\end{array}$ \\
\hline
\end{tabular}

$\star$ Definitions not identical but comparable.

$\star \star$ Recalculated from original data to allow comparison.

nurses. Mogensen (1970) found that approximately 50 per cent of ventricular tachycardias were detected on monitoring, in accordance with our findings. Since Mogensen reported only on the detection of ventricular tachycardia, comparisons regarding other types of ventricular arrhythmias are not possible.

We were especially interested in the delay between the appearance and detection of ventricular tachyarrhythmias, which has been reported to be alarmingly long (Romhilt et al., 1973; Vetter and Julian, 1975) (Table). The vast majority of arrhythmias detected by the nurses was discovered within the first half-hour period (Fig. 2-4). Furthermore, as can be seen from Fig. 6, warning and serious arrhythmias were detected in the first minute after their appearance in about 50 per cent of the arrhythmia-containing half-hour periods. This is shown by the fact that these arrhythmias were detected though they occurred during only one single minute of a half-hour period. The differing results in this and the previous reports can probably be explained by different standards of equipment and modes of operation of the CCUs. From our experience we have tried to identify factors of importance for the efficiency of arrhythmia detection.

The number of staff, their education, and the instrumental equipment are the most important factors that can be expected to influence the quality of electrocardiographic monitoring. Well-defined routines for observation and documentation of arrhythmias also help to keep the motivation for arrhythmia detection at a high level. In most units neither the size of the staff nor the design of the electrocardiographic monitoring equipment allows truly continuous electrocardiographic observation. Commonly, the cardiograms from all patients are displayed simultaneously only at a central station. The staff is often so small that the central station has to be left unmanned for periods of time during which the monitoring of the patients will be unsatisfactory. A system for decentralised electrocardiographic display allows the staff to leave the central station in order to attend one patient while still continuing the observation of the others. We think that the reliability of arrhythmia detection in our CCU results mainly from the use of a decentralised electrocardiographic display together with welldefined routines for observation and documentation of arrhythmias. Development along these lines may further increase the accuracy of arrhythmia detection. Whether this is of clinical value and worth the cost and effort (installation cost per bed is about £3750) can only be decided by comparing the cost efficiency of this system with that of others. As automated arrhythmia detection systems are now being developed comparisons of this kind become increasingly important.

The clinical significance of different types of ventricular tachyarrhythmias is still debated (Lown et al., 1967; Lie et al., 1974). In the elegant work by Lie et al. (1974) there is unfortunately no exact description of the detection rate of different arrhythmias. Though their results are interesting, some warning arrhythmias may not have been detected because the period of observation was too short. As the authors themselves pointed out, 5 out of 9 patients showing no warning arrhythmias before ventricular fibrillation had been monitored for only 10 to 30 minutes. Ventricular tachycardia and $R$ on $T$ VPCs are believed to be ominous signs of impending ventricular fibrillation (Mogensen, 1970; Dhurandhar et al., 1971; MacMillan and Brown, 1971; Bruyneel and Opie, 1973), while frequent unifocal VPCs are now thought to be less significant. If this is so, the value of electrocardiographic monitoring as presented here may be questioned. However, recent observations suggest that $R$ on $T$ VPCs and ventricular tachycardia together with paired VPCs and multifocal VPCs coexist with frequent unifocal VPCs in the vast 
majority of cases (Rydén et al., 1975a). If this is true, the easily detectable repetitive ventricular arrhythmias could be used to identify a group of patients prone to develop more malignant arrhythmias.

\section{References}

Bruyneel, K. J. J., and Opie, L. H. (1973). The value of warning arrhythmias in the prediction of ventricular fibrillation within one hour of coronary occlusion. Experimental studies in the baboon. American Heart fournal, 86, 373-384.

Christiansen, I., Iversen, K., and Skouby, A. P. (1970). Nytten afet koronarafsnitt. En sammenlignende undersфgelse. Ugeskrift for Laeger, 132, 1603-1610.

Dhurandhar, R. W., MacMillan, R. L., and Brown, K. W. G. (1971). Primary ventricular fibrillation complicating acute myocardial infarction. American fournal of Cardiology, 27, 347-351.

Henning, R., and Holmberg, S. (1971). Erfarenheter från Sahlgrenska sjukhusets hjärtinfarktavdelning. Läkartidningen, 68, 3603.

Hofvendahl, S. (1971). Influence of treatment in a coronary care unit on prognosis in acute myocardial infarction. A controlled study in 271 cases. Acta Medica Scandinavica, Suppl. 519.

Lie, K. I., Wellens, H. J., van Capelle, F. J., and Durrer, D. (1974). Lidocaine in the prevention of primary ventricular fibrillation. New Zealand fournal of Medicine, 291, 13241326.

Lown, B., Vassaux, C., Hood, W. B., Fakhro, A. M., Kaplinsky, E., and Roberge, G. (1967). Unresolved problems in coronary care. American fournal of Cardiology, 20, 494-508.
MacMillan, R. L., and Brown, K. W. G. (1971). Comparison of the effects of treatment of acute myocardial infarction in a coronary unit and on a general medical ward. Canadian Medical Association fournal, 105, 1037-1040.

Meltzer, L. E., and Kitchell, J. R. (1972). The development and current status of coronary care. In Textbook of Coronary Care. Ed. by L. E. Meltzer and A. J. Dunning. Charles Press, Philadelphia.

Mogensen, L. (1970). Ventricular tachyarrhythmias and lignocaine prophylaxis in acute myocardial infarction. Acta Medica Scandinavica, Suppl. 513.

Romhilt, D. W., Bloomfield, S. S., Chou, T.-C., and Fowler, N. O. (1973). Unreliability of conventional electrocardiographic monitoring for arrhythmia detection in coronary care units. American fournal of Cardiology, 31, 457-461.

Rydén, L., Hjalmarson, Å., and Waldenström, A. (1975a). Effects of the quarternary ammonium compound QX-572 on ventricular tachyarrhythmias complicating acute myocardial infarction. British Heart fournal, 37, 426-437.

Rydén, L., Waldenström, A., and Holmberg, S. (1975b). The reliability of intermittent ECG sampling in arrhythmia detection. Circulation, 52, 540-545.

Rydén, L., Waldenström, A., Ehn, L., Holmberg, S., and Husaini, M. (1973). Comparison between effectiveness of intramuscular and intravenous lignocaine on ventricular arrhythmia complicating acute myocardial infarction. British Heart fournal, 35, 1124-1131.

Vetter, N. J., and Julian, D. G. (1975). Comparison of arrhythmia computer and conventional monitoring in coronary-care unit. Lancet, 1, 1151-1154.

Requests for reprints to Dr. Stig Holmberg, Department of Medicine I, Division of Cardiology, Sahlgren's Hospital, S-413 45 Göteborg, Sweden. 